\title{
Efecto de diferentes proporciones de Tagetes minuta y Glycine max sobre Meloidogyne incognita
}

\author{
Susana A. SuáreZ ${ }^{1, \infty}$; Alejandra GiL²; Elba. de la Fuente²; María del C. Tordable ${ }^{1}$ \& \\ Claudio M. Ghersa ${ }^{3}$
}

\begin{abstract}
${ }^{1}$ Departamento de Ciencias Naturales, Universidad Nacional de Río Cuarto. Río Cuarto, Argentina. ${ }^{2}$ Departamento de Producción Vegetal, Facultad de Agronomía, Universidad de Buenos Aires. Ciudad Autónoma de Buenos Aires, Argentina. ${ }^{3}$ IFEVA/CONICET, Facultad de Agronomía, Universidad de Buenos Aires. Ciudad Autónoma de Buenos Aires, Argentina.
\end{abstract}

\begin{abstract}
Resumen. Se estudió la infestación de raíces por Meloidogyne incognita (Kofoid and White) Chitwood, en mezclas de plantas de Glycine max (L.) Merr. y Tagetes minuta L. en distintas proporciones, por medio de un experimento diseñado para probar cómo huéspedes en diferentes proporciones modifican la reproducción de la primera generación y la densidad de la segunda generación del nematodo. En ambos huéspedes se evaluó la densidad de agallas radicales, sitios de alimentación, reacciones histológicas y número de larvas de la segunda generación de $M$. incognita. La densidad de agallas fue mayor en los tratamientos donde las plantas de G. max se encontraban en más de $50 \%$. En raíces de T. minuta se encontraron sitios de alimentación no funcionales y las larvas en estos sitios no alcanzaron la etapa reproductiva. La reducción de la densidad de las larvas en el suelo fue proporcional a las cantidades de T. minuta en la mezcla. Estos datos indican que T. minuta infectado por nematodos actúa como una "planta trampa" debido a la captura de algunos individuos, muchos de los cuales nunca alcanzan la madurez sexual, y por la reducción de la densidad de segunda generación de $M$. incognita.
\end{abstract}

[Palabras clave: histopatología, mecanismos de defensa, interacción parasito-huésped, sobrevivencia]

\begin{abstract}
Effect of different proportions of Tagetes minuta and Glycine max on Meloidogyne incognita. Root infestation by Meloidogyne incognita (Kofoid and White) Chitwood, in mixtures of Glycine max (L.) Merr. and Tagetes minuta L. was studied through an experiment designed to test how host different proportions influenced nematode reproduction and second generation density. Root-knot density, feeding sites, histological reactions and number of second generation M. incognita in both host species were evaluated. Gall density was higher in treatments where G. max plants were in more than $50 \%$. In roots of T. minuta feeding sites they were found nonfunctional and larvae on these sites did not reach reproductive stage. Density reduction of soil larvae was proportional to the amounts of $T$. minuta in the mixture. These data indicate that $T$. minuta infected by nematodes acts as a "trap-plant", capturing some individuals, many of which will never reach sexual maturity, and reducing second generation density.
\end{abstract}

[Key words: histopathology, mechanisms of defence, parasite-host interaction, survival]

\section{INTRODUCCIÓN}

Las interacciones entre los componentes de la comunidad cultivo-maleza y los organismos heterótrofos pueden afectar el ensamble tanto de las especies vegetales como el de los organismos del nivel trófico superior (Grover and Holt 1997; Goldberg 1999; Bezemer et al. 2005; Stephen et al. 2005; de la Fuente et al. 2014). Los nematodos fitoparásitos pueden afectar el crecimiento y la reproducción de las plantas (e.g., con frecuencia modifican las relaciones de competencia entre plantas, lo que puede en última instancia, influir en la dinámica de las poblaciones y comunidades) (Yeates and Bongers 1999; Berkelmans et al. 2003; Wang et al. 2007). Del mismo modo, las plantas en su coevolución con nematodos fitoparásitos desarrollaron mecanismos para prevenir o minimizar su efecto (Zacheo Editor asociado: Esteban Jobbágy

ssuarez@exa.unrc.edu.ar and Bleve-Zacheo 1995; De Moraes et al. 1998). Las defensas químicas, acumuladas en estructuras internas específicas de las plantas, pueden repeler o ser tóxicas para los nematodos fitoparásitos. Estas defensas también pueden ser liberadas como exudados de la raíz o como compuestos volátiles capaces de modificar el entorno externo. Esto afecta, directa o indirectamente, a otros organismos en la comunidad (Yeates 1999; Jacobs et al. 1994; Zacheo and Bleve-Zacheo 1995; Gil et al. 2002).

Tagetes minuta L. (Asteraceae), "chinchilla", produce terpenos y tiofenos, y por eso se la usa en la industria para la extracción de anillos aromáticos. Además, expresa una variación intraespecífica amplia en cuanto a su composición química y a cómo responde a la herbivoría; muestra distintos

Recibido: 25 de abril de 2016

Aceptado: 26 de diciembre de 2016 
mecanismos de defensa y de tolerancia a nematodos fitoparásitos (Jacobs et al. 1994; Gil et al. 2002). La mayor concentración de tiofenos se encuentra en la raíz (Sütfeld 1982; Gil et al. 2002), lo que sugiere que estos productos químicos actúan como exudados que son liberados en el ambiente del suelo. Aunque existen estudios que indican que $T$. minuta puede afectar de forma negativa a los nematodos endoparásitos, se han observado agallas en raíces de Tagetes spp., lo que demuestra que pueden ser infestados por varias especies de Meloidogyne (Doucet 1999; Wang et al. 2007). Algunos autores sugieren que Tagetes spp. actuaría como "planta trampa" de Meloidogyne spp. (Wang et al. 2007; Hooks et al. 2010). Aunque los enfoques experimentales han informado los modos de acción (es decir, intra o extra radical, físicos o químicos) existen controversias entre los estudios, y las descripciones histopatológicas de esta interacción todavía son escasas (Ploeg and Maris 1999; Wang et al. 2007; Piedra Buena et al. 2008).

Las "plantas trampa" pueden reducir las poblaciones de fitoparásitos a través de dos mecanismos: a) por atracción y por permitir la infestación por parte del nematodo, con su posterior eliminación por la muerte o cosecha dela planta, ob) por permitir la infestación, pero inhibir su posterior desarrollo y reproducción. Estas plantas podrían ser incluidas como parte de prácticas agronómicas para reducir los daños por nematodos fitoparásitos en cultivos susceptibles (Ploeg and Maris 1999; Wang et al. 2007; Hooks et al. 2010).

Los nematodos de la agalla (Meloidogyne spp.) tienen una gama de huéspedes amplia y afectan considerablemente las actividades agrícolas en todo el mundo ya que disminuyen el rendimiento de los cultivos y aumentan la incidencia dehongos, bacterias y enfermedades virales (Doucet 1999; Doucet et al. 2000; Trudgill and Blok 2001; Lorenzo et al. 2004). Meloidogyne incognita (Kofoid and Blanco) Chitwood es un endoparásito obligado que penetra en la raíz como juvenil vermiforme de segundo estadio (J2) (Dropkin and Nelson 1960; Jepson 1987). La formación de agallas es el resultado del crecimiento del nematodo, del desarrollo de su sitio de alimentación y de las reacciones de raíz (e.g., hiperplasia e hipertrofia celular) (Dropkin and Nelson 1960; Doucet et al. 2000; Lorenzo et al. 2004).

M. incognita afecta al cultivo de soja (Glycine $\max ($ L.) Merr.) en todo el mundo (Wrather et al. 2001; de la Fuente et al. 2011). Nuestra hipótesis sostiene que una mezcla de G. max y de plantas trampa como T. minuta podría reducir el daño del nematodo de la agalla en G. max, en función de la proporción de ambas especies de plantas en la mezcla. Teniendo en cuenta que T. minuta crece como maleza en cultivos G. max, para controlarlo es importante conocer la proporción de T. minuta que podría afectar la supervivencia y la densidad de $M$. incognita. El objetivo del presente trabajo es evaluar el efecto en diferentes proporciones de las raíces de Tagetes minuta y Glycine max en la reproducción de Meloidogyne incognita y la densidad de larvas infestantes (J2) en la segunda generación.

\section{Materiales y Métodos}

\section{Diseño experimental}

Sellevóa cabo un experimento en invernadero utilizando un modelo sustitutivo con cuatro repeticiones. El modelo sustitutivo incluye condiciones puras y mezclas (Radosevich et al. 2007). Las especies utilizadas fueron G. max (variedad Don Mario 4800 RR) susceptibles a M. incognita (Doucet et al. 2000), y T. minuta, semilla recolectada de una población natural de la Pampa Ondulada, Argentina (Suárez et al. 2015). La proporción de plantas de G. max y T. minuta fue variable, mientras que la densidad total de plantas se mantuvo constante en todos los tratamientos (Radosevich et al. 2007).

Ambas especies se cultivaron inicialmente en cámara de crecimiento, y se empleó suelo esterilizado (van Bruggen and Grunwald 1996). Con el fin de iniciar el experimento con un tamaño similar de plantas para ambas especies, se seleccionaron al azar plántulas con dos hojas para G. max y con cuatro hojas para T. minuta.

Las plántulas se trasplantaron a macetas con una mezcla de arena y tierra (Argiudol 60:40 $[1.3 \%$ C, $0.15 \%$ N, 33.96 ppm P]). A esa mezcla se la esterilizó con gas bromuro de metilo (70 $\mathrm{g} / \mathrm{m}^{2}$ ) para obtener suelo libre de nematodos. Tagetes minuta y G. max se plantaron en densidades de 8 plantas / maceta de $30 \mathrm{~L}(40 \mathrm{~cm}$ de diámetro), en condiciones puras y mixtas, con o $\sin M$. incognita. Los tratamientos fueron: G. max puro (100\%), T. minuta puro $(0 \%$ de $G$. max) y tres mezclas de 6, 4 y 2 plantas de $G$. max con 2, 4 y 6 plantas de T. minuta $(75 \%$, $50 \%$ y $25 \%$ de G. max). Las macetas se regaron una vez al día usando un sistema de riego por goteo automático, y se mantuvieron durante 12 semanas, entre enero y abril, después del trasplante, en condiciones de invernadero (17$27^{\circ} \mathrm{C}$, con luz natural). Una semana después 
del trasplante se añadieron 2100 J2 móviles de M. incognita a cada maceta del tratamiento con nematodos. El inoculo se colectó mediante el método de flotación-centrifugación (Jenkins 1964) a partir de plantas de tomate infestadas, cultivadas en un invernadero.

\section{Método de muestreo y procesamiento}

El muestreo de raíces G. max y T. minuta y del suelo se realizó 78 días después de inocular el suelo. Las raíces de cada especie se seleccionaron al azar y se fijaron en FAA (formol 10\%, alcohol etílico $96^{\circ} 50 \%$, ácido acético glacial 5\%, agua destilada 35\%). El ancho, el largo y el número de agallas se evaluó en cuatro submuestras. Cada submuestra de $8 \mathrm{~cm}$ de raíz se seleccionó al azar para formar una muestra de $32 \mathrm{~cm}$ de raíz por planta. Las agallas se contaron usando un microscopio estereoscópico Stemi SV6 Carl Zeiss con magnificación 1x.

Para el análisis histopatológico, diez muestras de raíces con y sin nematodos se cortaron en secciones de 3 a $4 \mathrm{~mm}$ de largo, y se fijaron en FAA durante al menos 48 horas. Las muestras se deshidrataron en una serie gradual de alcohol etílico y de xilol, y se incluyeron en cera histológica. Se obtuvieron cortes transversales seriados de raíz de 10 a 12 $\mu \mathrm{m}$ de espesor por medio de un micrótomo rotatorio. Luego, se tiñeron con hematoxilinasafranina-verde rápido y se montaron con Depex (O’Brien and Mc Cully 1981). Algunas secciones sin colorear se utilizaron en pruebas histo-químicas para identificar compuestos químicos en las paredes de las células gigantes, relacionados con la penetración y el movimiento de nematodos. Con el fin de detectar lignina y defensas físicas, las muestras se trataron con una solución de alcohol con $1 \%$ de floroglucinol HCL (O'Brien and Mc Cully 1981). Estos cortes se examinaron usando un microscopio de fluorescencia Axiophot Carl Zeiss equipado con lámparas de vapor de mercurio $100 \mathrm{~W}$ HBO y $50 \mathrm{~W}$, y BP 450-490 y filtros BP 365. Las microfotografías se tomaron con un microscopio Axiophot Carl Zeiss con cámara AxioCam HRc, y se digitalizaron con el software AxioVision 4.3. Larvas J2 de $M$. incognita se extrajeron de $200 \mathrm{~g}$ de suelo de cada maceta usando el método de flotacióncentrifugación (Jenkins 1964) y se contaron bajo un aumento $40 x$.

\section{Análisis de datos}

La relación entre la longitud y el ancho de las agallas se analizó mediante regresión con ajuste lineal (vers. Prisma 4, 2003). El valor observado de $M$. incognita (Mo) se calculó contando las larvas al final del experimento y se analizó mediante ANOVA y una prueba de Tukey $(P<0.01)$ (Montgomery 1991). El valor esperado $(\mathrm{Me})$ se estimó mediante la adición de la contribución de larvas de cada especie de la mezcla, lo que resultó de multiplicar el número de plantas de cada especie en la mezcla por Mo en la condición pura de G. max y T. minuta:

$\mathrm{Me}=$ planta de $\mathrm{G} \times$ Mo G + planta de $\mathrm{T} \times \mathrm{Mo} \mathrm{T}$

Mo $\mathrm{G}=$ número de larvas / 8 plantas de $\mathrm{G}$

Mo $\mathrm{T}$ = número de larvas $/ 8$ plantas de $\mathrm{T}$

Los valores medios observados y esperados de larvas $M$. incognita se analizaron mediante la prueba $\mathrm{t}$ de Student $(P<0.05)$. La relación entre la densidad de larvas y número de agallas con la proporción de G. max en la mezcla se analizó utilizando regresión lineal (vers. Prisma 4, 2003).

\section{Resultados}

\section{Agallas radicales}

La densidad de agallas fue mayor $(P<0.05)$ cuando el porcentaje de G. max en la mezcla era superior al 50\% (Figura 1). La longitud y el ancho de las agallas estuvieron linealmente relacionadas $G$. $\max \left(\mathrm{r}^{2}=0.64\right)$, T. minuta $\left(\mathrm{r}^{2}=0.53\right)$ $\mathrm{y}$ total $\left(\mathrm{r}^{2}=0.58\right)$ (Figura 2$)$, y las pendientes de las regresiones no fueron significativamente diferentes entre G. max y T. minuta $(P<0.1)$. La pendiente de la línea de regresión del total de las agallas fue significativamente distinta de cero $(P<0.0001)$ (Figura 2).

En ambas especies, infestadas por $M$. incognita, la hiperplasia celular y la hipertrofia fueron evidentes en las agallas. La hiperplasia afectó el cilindro central (Figuras 3B, 3G y 4B) y la hipertrofia generó células gigantes (Figuras 3B, 3G, 4B y 4G). Estos procesos redujeron y desorganizaron los tejidos vasculares, alterando la posición de las células del xilema (Figuras 3A, 3B, 3G y 4A, 4B, 4C y 4G).

Los sitios de alimentación funcionales estaban formados por 6 a 10 células gigantes (Figuras 3, 4G y 4D), con un tamaño promedio de $190 \mu \mathrm{m}$ (medida a lo largo de eje mayor de la célula). Las células gigantes presentaron citoplasma denso y múltiples núcleos hipertróficos. Estos núcleos se organizaban en grupos o dispersos en el citoplasma (Figuras 3C, 3D, 4D y 4E). Las paredes de las células gigantes adyacentes al miembro de vasos del xilema (célula 


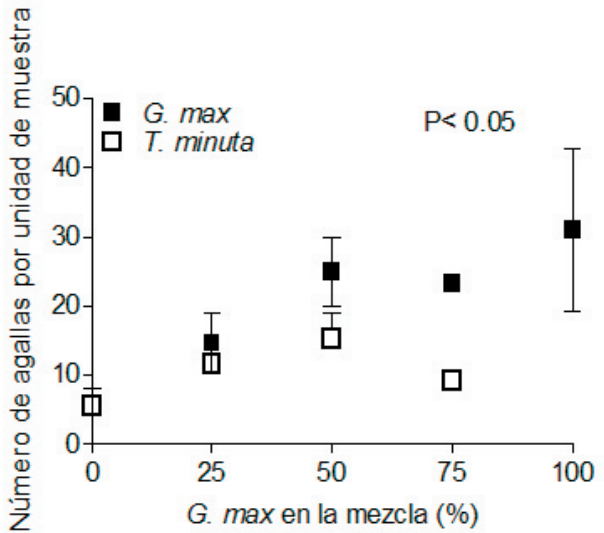

Figura 1. Número de agallas en raíces de G. $\max$ y $T$. minuta en relación con la proporción de plantas de $G$. max, en la mezcla de plantas de G. max y T. minuta. Se muestra el valor de probabilidad estimada por el modelo de regresión lineal.

Figure 1. Number of galls on roots of G. max and T. minuta in relation to the proportion of G. max plants, in the mixture of G. max and T. minuta plants. Probability value estimated by linear regression model shown.

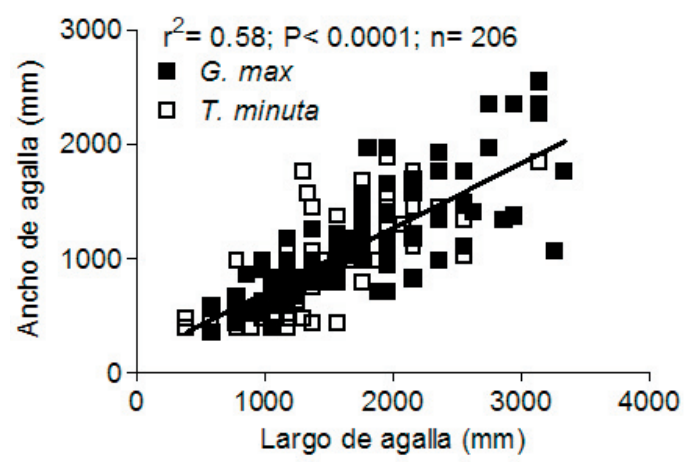

Figura 2. Relación entre largo y ancho de las agallas en las raíces de G. max y T. minuta infectadas por M. incognita. La línea indica el ajuste del modelo de regresión lineal y el valor de probabilidad estimada para el total de las agallas $(n=206)$.

Figure 2. Relationship between length and width of galls on roots of G. max and T. minuta infected by M. incognita. The line indicates the linear regression model fit and the probability value estimated for total galls $(n=206)$.

conductora) eran de $8 \mu \mathrm{m}$ en promedio con invaginaciones y de características celulósicas (Figuras 3C, 4D y 4E).

\section{Sobrevivencia y reproducción de $M$. incognita}

En ambas especies vegetales, las masas de huevos se observaron asociadas a hembras con los sitios de alimentación funcionales (Figuras 3B y 4B). Dos sitios de alimentación funcionales se observaron por sección de agalla en G. max (Figura 3G). En tanto que, sitios de alimentación no funcionales se observaron en T. minuta (Figura 4G). Las células gigantes que formaban estos sitios estaban muertas e invadidas por células parenquimáticas. El espesor de las paredes celulares era de $6 \mu \mathrm{m}$ en promedio y conservaban su característica celulósica (Figura 4G).

Se observaron Larvas (J1) en el interior de los huevos y larvas (J2) en la masa de huevos junto al mucílago en G. max (Figura 3E y 3F). En T. minuta, las masas de huevos estaban cubiertas por mucílago fibroso y algunos huevos estaban plamolizados (Figura 4F). La densidad de J2 en el suelo fue significativamente mayor en G8/T0 que en G0/T8 $(P<0.01)$ (Figura 5).

La abundancia de larvas de $M$. incognita difirió significativamente entre tratamientos $(P=0.004)$. El análisis de los valores medios separó el tratamiento G. max $100 \%$ de los tratamientos G. $\max 25 \%$ y $0 \%$ (Tabla 1 ). La hipótesis de igualdad entre la media de las larvas esperada y observada para los tratamientos G. $\max 75 \%, 50 \%$ y $25 \%$ no pudo ser rechazada $(P<0.05)$.

Tabla 1. Abundancia promedio de larvas de M. incognita al final de experimento en $200 \mathrm{~g}$ de suelo.

Table 1. Average abundance of $M$. incognita larvae at the end of the experiment in $200 \mathrm{~g}$ of soil.

\begin{tabular}{|c|c|c|c|c|c|c|}
\hline \multirow{2}{*}{$\begin{array}{l}\text { Meloidogyne } \\
\text { incognita } \\
\text { ( } n^{\circ} \text { de larvas) }\end{array}$} & \multicolumn{5}{|c|}{ Glycine max en la mezcla (\%) } & \multirow[t]{2}{*}{$P$} \\
\hline & 100 & 75 & 50 & 25 & 0 & \\
\hline \multirow[t]{2}{*}{ Observado } & 121 & 69 & 89 & 34 & 10 & \\
\hline & a & $\mathrm{abc}$ & $\mathrm{ab}$ & $\mathrm{bc}$ & $\mathrm{c}$ & $<0.01$ \\
\hline \multirow[t]{2}{*}{ Esperado } & 121 & 93 & 65 & 38 & 10 & \\
\hline & & $\mathrm{ns}$ & ns & ns & & $<0.05$ \\
\hline
\end{tabular}

Letras distintas indican diferencias significativas entre las medias observadas según prueba de Tukey $(P<0.01)$; ns indica diferencia no significativa entre medias observadas y esperadas según prueba de Student $(P<0.05)$.

Different letters indicate significant differences between means observed according Tukey test $(P<0.01)$; ns: indicates no significant differences between means observes and expected according Student test $(P<0.05)$.

\section{Discusión}

$\mathrm{Al}$ igual que en estudios anteriores (Jepson 1987; Doucet 1999; Trudgill and Blok 2001; Wang et al. 2007), los resultados indican que tanto en las raíces de G. $\max$ como en las de T. minuta se forman agallas; esto indica que $M$. incognita parasita ambas especies. Sin embargo, cada especie posee diferentes mecanismos internos y externos a la raíz que afectan de forma diferencial a la primera y a la segunda generación del nematodo de la agalla (Figura 6). 

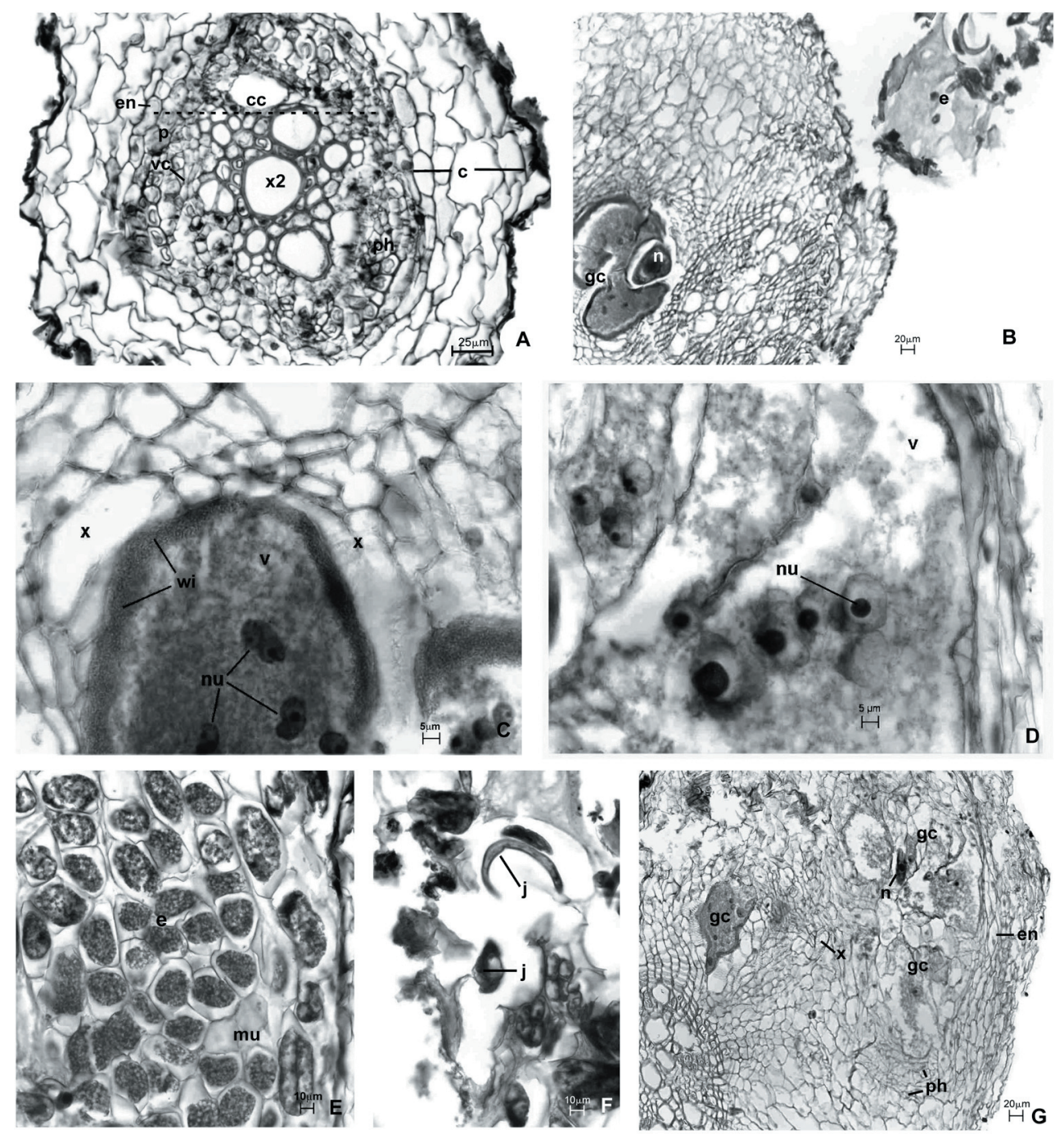

Figura 3. Microfotografías de cortes transversales de raíces de G. max. A) Raíz sin infectar por M. incognita. B) Agalla con nematodo, sitio de alimentación en el cilindro central y masa de huevo con larva. C- F) Detalles. C) Características citológicas y de la pared de una célula gigante. D) Núcleos en una célula gigante. E) Huevos en mucílago. F) J2 en masa de huevos. G) Sector de agalla con dos sitios de alimentación.

Abreviaturas. c: corteza; cc: cilindro central; e: huevos; en: endodermis; gc: célula gigante; j: larva; mu: mucílago; n: nematodo; nu: núcleo; p: periciclo; ph: floema; v: vacuola; vc: cambium vascular; wi: pared celular con invaginaciones; $\mathrm{x}$ : xilema; $\mathrm{x2}$ : xilema secundario.

Figure 3. Photomicrographs of cross-sections of G. max roots. A) Root non-infected by M. Incognita. B) Gall with nematode, feeding site in central cylinder and egg mass with larvae on the surface. C- F) Details. C) Wall and cytological characteristics of a giant cell. D) Nucleus in a giant cell. E) Eggs in mucilagus. F) J2 in egg mass. G) Sector of gall with two feeding sites.

Abbreviations. c: cortex; cc: central cylinder; e: eggs; en: endodermis; gc: giant cells; j: larva; mu: mucilagus; n: nematode; nu: nucleus; p: pericycle; ph: phloem; v: vacuole; vc: vascular cambium; wi: cell wall with wall ingrowths; x: xylem; $\mathrm{x} 2$ : secondary xylem

El menor número de agallas en las mezclas de ambas especies respecto a la condición pura de G. max indica que las variaciones en la proporción de T. minuta podrían generar diferentes oportunidades para que los nematodos encuentren la raíz más adecuada para parasitar. Por otro lado, las diferencias en el número de agallas entre las condiciones puras indican una preferencia por una de las especies huésped. Como lo indican otros estudios, las características químicas del ambiente rizosférico de G. max y de T. minuta podrían afectar las preferencias de infestación (Jacobs et al. 1994; Gil et al. 2002).

Aunque se utiliza para evaluar la susceptibilidad o la resistencia de la planta a los nematodos de la agalla según el número 

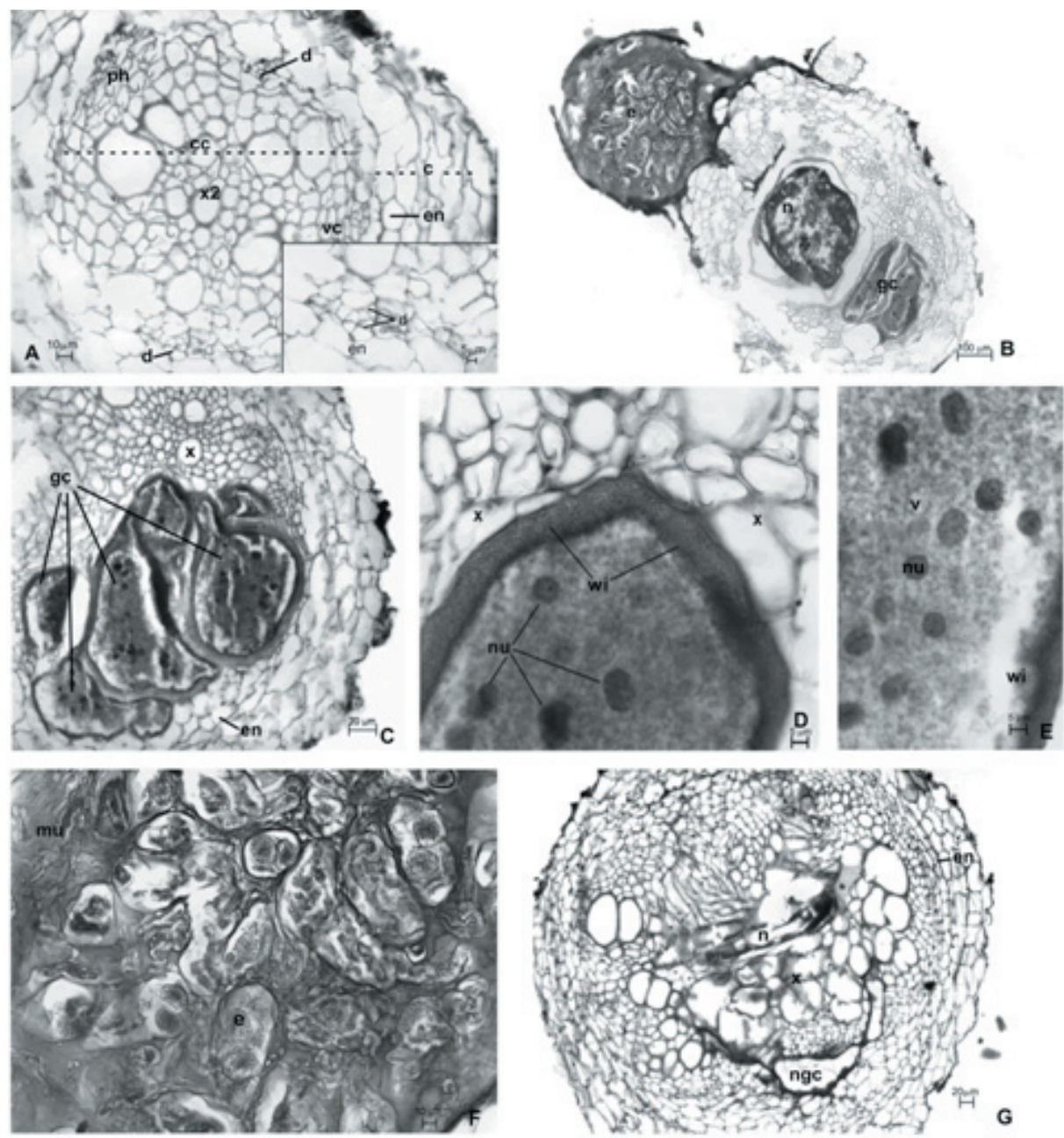
Figure 4. Microfotografías de cortes transversales por raíces de T. minuta. A) Raíz sin infectar por M. incognita, con
detalle donde se muestra un conducto. B) Agalla con nematodo y sitio de alimentación en cilindro central y masa de huevo externa. C) Cilindro central con sitio de alimentación. D-F) Detalles. D) Características citológicas de una célula gigante. E) Pared celular con invaginaciones. F) Huevos en mucílago. G) Agalla con nematodo y sitio de alimentación no funcional.

Abreviaturas. c: corteza; cc: cilindro central; d: conducto; e: huevos; en: endodermis; gc: célula gigante; mu: mucilago; n: nematodo; ngc: célula gigante no-funcional; nu: núcleo; ph: floema; v: vacuola; vc: cambium vascular; wi: pared celular con invaginaciones; $x$ : xilema; $x 2$ : xilema secundario.

Figure 4. Photomicrographs of cross-sections of T. minuta roots. A) Root non-infected by M. Incognita, with detail showing duct. B) Gall with nematode and feeding site in the central cylinder and egg mass on the surface. C) Central cylinder with feeding site. D- F) Details. D) Cytological characteristics of a giant cell. E) Cell wall (positive PAS) showing wall ingrowths. F) Eggs in mucilagus. G) Gall with nematode and non-functional feeding site.

Abbreviations. c: cortex; cc: central cylinder; d: duct; e: eggs; en: endodermis; gc: giant cells; mu: mucilagus; n: nematode; ngc: non-functional giant cells; nu: nucleus; ph: phloema; v: vacuole; vc: vascular cambium; wi: cell wall with wall ingrowths; $x$ : xylem; $x 2$ : secondary xylem.

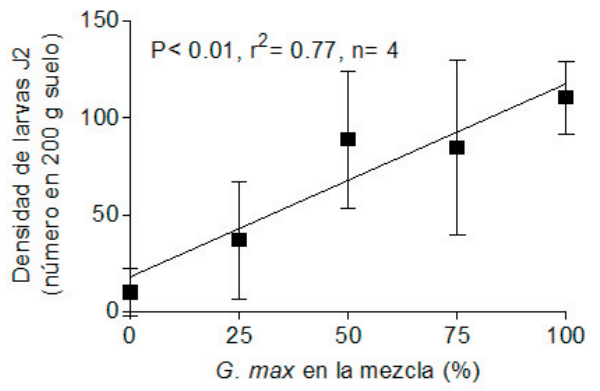

Figura 5. Relación entre la densidad de larvas infestantes (J2) y la proporción de plantas de G. max en la mezcla de plantas de G. max y T. minuta. La línea indica el ajuste del modelo de regresión lineal y el valor de probabilidad estimada para la densidad de larvas en las distintas proporciones de plantas en las mezclas $(n=4)$.

Figure 5. Relationship between the density of infective larvae (J2) and the proportion of G. max plants, in mixture of G. $\max$ and T. minuta plants. The line indicates the linear regression model fit and the probability value estimated for density of larvae in different proportions in the mixtures of plants $(n=4)$. 


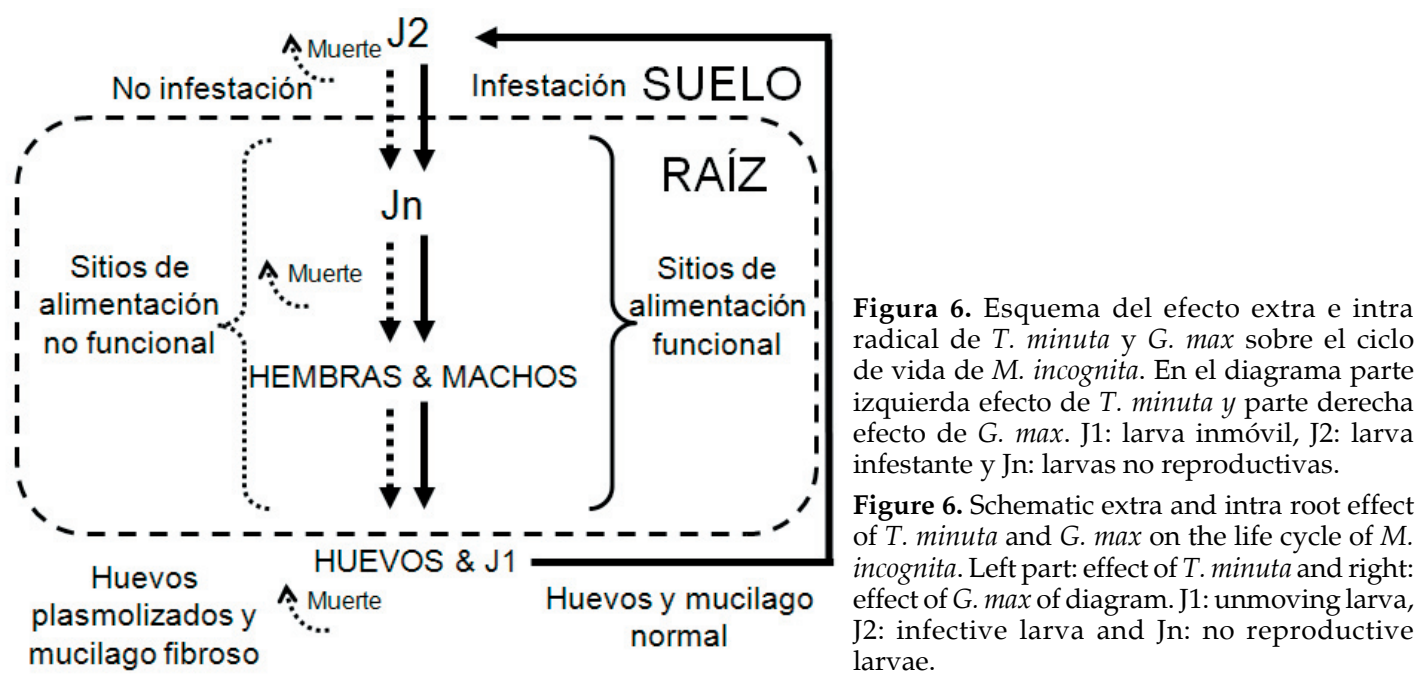

y el tamaño de las agallas (Gilli et al. 2003), nosotros consideramos que la utilización de estos parámetros para clasificar especies de planta como susceptible, resistentes o tolerantes a los nematodos no es suficiente. En concordancia con ello, otros autores proponen que las categorías se evalúen teniendo en cuenta el efecto de la planta en el nematodo y sus descendientes (e.g., análisis histopatológico) (Dropkin and Nelson 1960; Baker 1993) y la respuesta de la planta al nematodo (e.g., la producción de biomasa) (Wang et al. 2007; Wrather et al. 2001).

En concordancia con otros estudios (Zacheo and Bleve-Zacheo 1995; Doucet et al. 2000), la hiperplasia celular aparece como mecanismo defensivo. No obstante, ninguna de las especies vegetales mostró defensas físicas tales como depósitos extra de lignina o de suberina (Baayen et al. 1996) ni respuesta necrótica, como se ha informado para T. patula (Piedra Buena et al. 2008). Los conductos secretores producen y conducen tiofenos y se relacionan con las defensas químicas (Vasudevan et al. 1997; Simon et al. 2002). En este trabajo, tales conductos se encontraron en T. minuta. Las células gigantes en G. max y T. minuta fueron similares a las descritas para otros cultivares de G. max infectados con diferentes especies de Meloidogyne (Dropkin and Nelson 1960; Doucet et al. 2000; Trudgill and Blok 2001). Las invaginaciones de las paredes de células gigantes indican una buena relación parásitohuésped ya que aumentan la superficie por la cual los productos metabólicos producidos por el huésped son constantemente removidos por el parásito (Zacheo and Bleve-Zacheo 1995).

Las diferencias entre los sitios de alimentación funcionales y no funcionales en
T. minuta fueron similares a los identificados por Doucet et al. (2000), Dropkin and Nelson (1960) y Lorenzo et al. (2004). Sin embargo, sus descripciones de sitios de alimentación no funcionales están relacionadas con hembras de nematodos que finalizaron su ciclo de vida, mientras que aquí, estos sitios invadidos por células parénquimáticas se relacionan con larvas muertas antes de la etapa reproductiva. Los dos sitios de alimentación funcionales observados en las secciones de agallas de G. max indican el establecimiento de más de una larva de $M$. incognita por agalla, en concordancia con Doucet et al. (2000) y Lorenzo et al. (2004).

La contribución de las larvas de M. incognita a la siguiente generación se modificó por la proporción de cada especie vegetal en la mezcla. Glycine max tenía más agallas que $T$. minuta, y es probable que cada agalla fuera el resultado de más de un sitio de alimentación. Por lo tanto, el número de larvas procedentes de G. max podría contrarrestar la reducción de las larvas promovido por T. minuta en mezclas. Esto podría explicarse por un efecto nematicida directo en los huevos (Siddiqui and Mashkoor-Alma 1988; Ploeg 1999, 2002).

Los resultados de este estudio mostraron los mecanismos internos y externos de la raíz que explican el efecto de $T$. minuta sobre M. incognita. Estos resultados permiten la reinterpretación de investigaciones anteriores y proporcionan elementos para postular explicaciones funcionales integrales (Figura 6). Meloidogyne incognita puede completar su ciclo de vida y producir huevos en ambas especies, pero en T. minuta, el crecimiento de la población será menor que en G. max. Además, en T. minuta, aunque algunos de los 
nematodos ingresan a la raíz, no establecen sitios de alimentación. Por lo tanto, T. minuta podría actuar como una "planta trampa" por capturar individuos dentro de la raíz (Hooks et al. 2010); muchos de éstos nunca alcanzarán la madurez sexual. También se observó que se reduce el número de J1 y J2 de la segunda generación por modificaciones en el mucílago que protege los huevos de la desecación. De esta manera, mediante la disminución de nematodos parásitos para producir descendencia y de su descendencia, T. minuta puede reducir la infestación a corto y largo plazo sobre otros individuos de la misma o de otras especies de la comunidad vegetal. El estudio histopatológico de la raíz es importante para entender qué factores son responsables del aumento y reducción en la supervivencia y la reproducción de nematodos. Teniendo en cuenta que la reducción en la densidad de las larvas es proporcional al número de plantas de T. minuta en la mezcla, esta especie podría ser utilizada en cultivos de cobertura o en intercultivos, como cultivo productor de aceite esencial, para obtener un beneficio extra.

AgradecimiEnTos. Los autores agradecen al Dr. M. E. Doucet, por corroborar la identidad de Meloidogyne incognita, y a los revisores anónimos, por sus sugerencias. Este trabajo fue financiado por CONICET, FONCyT, Universidad de Buenos Aires y SECyT de la Universidad Nacional de Río Cuarto.

\section{REFERENCIAS}

Baayen, R. P., G. B. Oullette, and D. Rioux. 1996. Compartmentalization of decay in carnations resistant to Fusarium oxysporum f. sp. dianthi. Phytopathology 86(10):1018-1031.

Baker, K. R. 1993. Resistance / tolerance and related concepts / terminology in plant nematology. Plant Disease 77(2): 111-113.

Berkelmans, R., H. Ferris, M. Tenuta, and A. H. C. van Bruggen. 2003. Effects of long-term crop management on nematode trophic levels other than plant feeders disappear after 1 year of disruptive soil management. Applied Soil Ecology 23:223-235.

Bezemer, T. M., G. B. De Deyn, T. M. Bossinga, N. M. van Dam, J. A. Harvey, and W. H. Van der Putten. 2005. Soil community composition drives aboveground plant-herbivore, parasitoid interactions. Ecology Letters 8:652-661.

De Moraes, C. M., W. J. Lewis, P. W. Paré, H. T. Alborn, and J. H. Tumlinson. 1998. Herbivore-infested plants selectively attract parasitoids. Nature 393:570-573.

Doucet, M. E. 1999. Nematodos del suelo asociados con vegetales en la República Argentina. Academia Nacional de Agronomía y Veterinaria, Series 24. Buenos Aires, Argentina. Pp. 259.

Doucet, M. E., S. A. Suárez, and E. Lorenzo. 2000. Alteraciones causadas por una población de nematodo fitófago del género Meloidogyne en un cultivar de soja, proveniente de Córdoba, Argentina. Kurtziana 28(2):221-228.

Dropkin, V.H., and P. E. Nelson. 1960. The histopathology of root-knot nematode infections in soybeans. Phytopathology 50:442-449.

de la Fuente, E. B., S. A. Suárez, A. Lenardis, and S. L. Poggio. 2014. Intercropping sunflower and soybean in intensive farming systems: evaluating yield advantage and effect on weed and insect assemblages. NJAS, Wageningen Journal of Life Sciences. Pp. 47-52.

de la Fuente, E. B., A. Gil, S. A. Suárez, C. M. Morvillo, and F. H. Oreja. 2011. Interacciones entre el cultivo de soja y los organismos que lo acompañan: efectos del manejo. Pp. 143-148 en O. S. Correas, E. B. de la Fuente, M. A. Carmona, A. G. Kantolic, and R. S. Lavado (compiladores). Soja: investigación científico-técnica desarrollada en INBA (CONICET/ FAUBA) y en la Facultad de Agronomía de la UBA. Editorial Facultad de Agronomía (UBA).

Gil, A., C. M. Ghersa, and S. Perelman. 2002. Root thiophenes in Tagetes minuta L. accessions from Argentina: genetic and environmental contribution to changes in concentration and composition. Biochemical Systematics and Ecology 30: $1-13$.

Gilli, J. R., D. Croatto, H. Baigorri, and L. Gadbán. 2003. Resistencia genética en genotipos comerciales de soja frente al nematodo de la agalla Meloidogyne spp. Córdoba (Argentina). Instituto Nacional de Tecnología Agropecuaria, Marcos Juárez, Argentina.

Goldberg, D. E. 1999. Components of resource competition in plant communities. Pp. 27-49 en D. Tilman and J. H. Grace (eds.). Perspectives on plant competition. Academic Press Inc.

Grover, J. P., and R. D. Holt. 1997. Plants in trophic webs. Pp. 556-567 en M. J. Crawley (ed.). Plant ecology. Blackwell Sicence Ltd., Oxford, USA.

Hooks, C. R. R., K-H. Wang, A. Ploeg, and R. McSorley. 2010. Using marigold (Tagetes spp.) as a cover crop to protect crops from plant-parasitic nematodes. Applied Soil Ecology 46:307-320.

Jacobs, J. J. M. R., A. Engelberts, A. F. Croes, and G. J. Wullems. 1994. Thiophene synthesis and distribution in young developing plants of Tagetes patula and Tagetes erecta. Journal Experimental Botany 45:1459-1466.

Jenkins, W. R. 1964. A rapid centrifugal-flotation technique method for separating nematodes from soil. Plant Disease Reporter 18:602.

Jepson, S. D. 1987. Identification of root-knot nematodes (Meloidogyne species). Farnham Royal, CAB International. London, UK. Pp. 265.

Lorenzo, E., M. E. Doucet, S. A. Suárez, and P. Lax. 2004. Histopathology of Melilotus albus roots parasitized by 
Meloidogyne javanica. Nematología Brasileira 28(1):111-114.

Montgomery, D. C. 1991. Diseño y análisis de experimentos. Grupo editorial Iberoamérica, Bs.As., Argentina.

O'Brien, T. P., and M. E. Mc Cully. 1981. The study of plant structure: principles and selected methods. Termacarphi Pty Ltd. Melbourne, Australia. Pp. 339.

Piedra Buena, A., M. Á. Diez-Rojo, J. A. López-Pérez, L. Robertson, M. Escuer, and A. Bello. 2008. Screening of Tagetes patula L. on different populations of Meloidogyne. Crop Protection 27:96-100.

Ploeg, A. T. 1999. Greenhouse studies on the effect of marigold (Tagetes spp.) on four Meloidogyne species. Journal Nematology 31(1):62-69.

Ploeg, A. T. 2002. Effects of selected marigold varieties on root-knot nematodes and tomato and melon yields. Plant Disease 86(5):505-508.

Ploeg, A. T., and P. C. Marüs. 1999. Effect of temperature on suppression of Meloidogyne incognita by Tagetes cultivars. Supplement to the Journal Nematology 31:709-714.

Radosevich, S., J. Holt, and C. M. Ghersa. 2007. Plant-plant associations. 6. Pp. 183-258 en S. Radosevich, J. Holt and C. M. Ghersa (eds.). Ecology of weeds and invasive plants. Relationship to agriculture and natural resources management. John Wiley \& Sons, Inc. New Jersey, USA.

Siddiqui, M. A., and M. N. Mashkoor-Alma. 1988. Control of plant parasitic nematodes by Tagetes tenuifolia. Revue de Nématologie 11:369-370.

Simon, P. M., L. Katinas, and A. M. Arambarri. 2002. Secretory structures in Tagetes minuta (Asteraceae, Helenieae). Boletín de la Asociación Argentina de Botánica 37(3-4):181-191.

Suárez, S. A., E. B. de la Fuente, A. Lenardis, A. Gil, M. E. Doucet, and C. M. Ghersa. 2015. Efecto de los factores de manejo agrícola del cultivo de soja sobre las malezas y su importancia sobre los grupos funcionales de nematodos edáficos en la Pampa Ondulada. Agronomía y Ambiente 35(1):71-81.

Stephen, H. T., J. Schroeder, and L. W. Murray. 2005. The role of weeds in nematode management. Weed Science 53: 923-928.

Sütfeld, R. 1982. Distribution of thiophene derivatives in different organs of Tagetes patula seedings grown under various conditions. Planta 156:536-540.

Trudgill, D. L., and V. Blok. 2001. Apomictic, polyphagous root-knot nematodes: exceptionally successful and damaging biotrophic root pathogens. Annual Review of Phytopathology 39:53-77.

van Bruggen, A. H. C., and N. J. Grunwald. 1996. Test for risk assessment of root infection by plant pathogens. 49. Pp. 293-310 en J. W. Doran and A. J. Jones (eds.). Methods for assessing soil quality. Soil Science Society of America. Madison, USA.

Vasudevan, P., S. Kashyap, and S. Sharma. 1997. Tagetes: a multipurpose plant. Bioresource Technology 62:29-35.

Wang, K-H., C. R. Hooks, and A. Ploeg. 2007. Protecting crops from nematode pests: using Marigold as an alternative to chemical nematicides. Plant Disease 35:1-6.

Wrather, J. A., T. R. Anderson, D. M. Arsyad, Y. Tan, L. D. Ploper, A. Porta-Puglia, H. H. Ram, and J. T. Yorinori. 2001. Soybean disease loss estimates for the top ten soybean-producing countries in 1998. Canadian Journal Plant Pathology 23:115-121.

Yeates, G. W. 1999. Effects of plants on nematode community structure. Annual Review of Phytopathology 37:127149.

Yeates, G. W., and T. Bongers. 1999. Nematode diversity in agroecosystems. Agriculture, Ecosystems and Environment 74:113-135.

Zacheo, G, and T Bleve-Zacheo. 1995. Plant-nematode interactions: histological, physiological and biochemical interactions. Pp. 321-353 en K. Kohmoto, U. S. Singh and R. P. Singh (eds.). Pathogenesis and host specificity in plant diseases. Histological, Biochemical, Genetic and molecular bases. Eukaryotes Pergamon, Elsevier Science Ltd. London, Great Britain. 